\title{
ESDA2010-25266
}

\section{MODELING OF NON-NEWTONIAN FLUID FLOW WITHIN SIMPLEX ATOMIZERS}

\author{
Mohammad Rezaeimoghaddam \\ Graduate student \\ Department of Mechanical Engineering \\ Ferdowsi University \\ Mashhad, Iran, \\ Email:m.rezaei.moghaddam@gmail.com \\ M. R. Modarres Razavi \\ Professor \\ . Department of Mechanical Engineering \\ Ferdowsi University \\ Mashhad, Iran \\ Email:m-razavi@um.ac.ir
}

\author{
Rasool Elahi* \\ Graduate student \\ Department of Mechanical Engineering \\ Ferdowsi University \\ Mashhad, Iran \\ Email:r.elahi.msc@gmail.com \\ Mohammad B. Ayani \\ Assistant Professor \\ Department of Mechanical Engineering, \\ Ferdowsi University \\ Mashhad, Iran \\ Email:mbayani@um.ac.ir
}

\begin{abstract}
In this paper the Volume-of-Fluid (VOF) method is used to simulate Newtonian and non-Newtonian fluid flow within simplex (pressure-swirl) atomizers. The two-dimensional axisymmetric swirl Navier-Stokes equations coupled with the VOF method is employed for accounting the formation mechanism of the liquid film inside the swirl chamber and the orifice hole of the pressure swirl atomizer. For verification of the code, the numerical results were compared with experimental data for large scale prototype injector with water (Newtonian fluid) as injection fluid in various constant inlet mass flow rate. By using power-law equation to calculate shear stress terms in the Navier-Stokes equations, the code is extended to compute Newtonian and non-Newtonian fluid flow inside the atomizer. The time-independent purely viscous power-law fluids flow in pressure-swirl atomizers is simulated. The effects of shear-thinning fluids $(0.5<n<1)$, viscous Newtonian $(n=1)$ fluids and shear thickening fluids $(1<n<$ 1.5) on atomizers performance (discharge coefficient and spray cone angle) were investigated. Results were shown that with increasing the power-law index the spray cone angle decreases and the discharge coefficient increases.
\end{abstract}

\section{INTRODUCTION}

Simplex atomizers are widely used in air-breathing gas turbine and combustion as they provide good spray and also they are relatively simple and inexpensive to manufacture [1]. In spite of widely used simplex atomizers involving Newtonian fluids, there are many applications that use the non-Newtonian fluids as injection liquid in simplex injectors such as pharmaceutical products, paint sprays, spray-drying of food and agriculture sprays. The atomizer's principle of operation is simple. It consists of the inlet grooves, swirl chamber and discharge orifice (see Fig. 1). Liquid is fed into the swirl chamber through tangential ports. The liquid is accelerated through the swirl chamber and then enters the orifice hole. The swirl motion of the liquid pushes it close to the wall and creates a zone of low pressure along the center line which results in back flow of air in the injector. The liquid emerges from the orifice as a conical sheet that spreads radially outwards due to centrifugal force.

\footnotetext{
*Graduate student and author of correspondence
} 

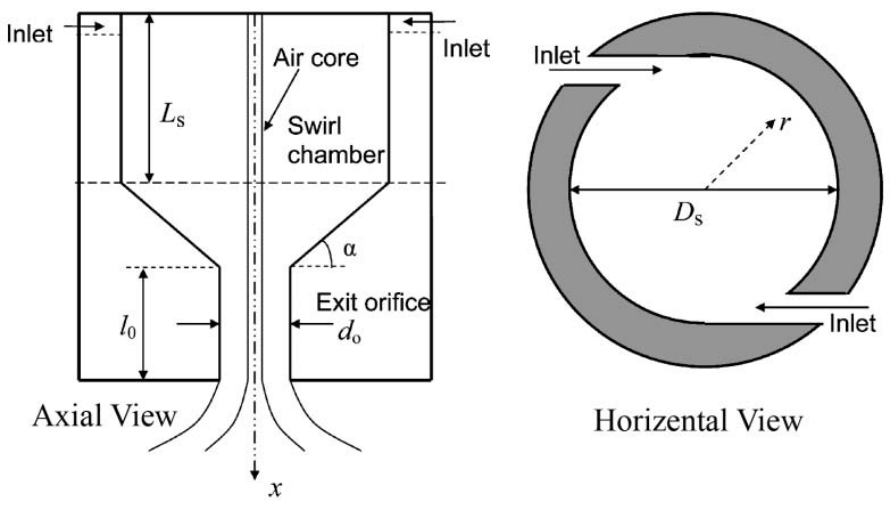

Horizental View

Figure1: SCHEMATIC VIEW OF A PRESSURE-SWIRL INJECTOR [20].

Due to the tangential velocity of flow inside the injector, an aircored vortex flow of liquid takes place in the nozzle and the liquid comes out of the orifice in the form of a thin film. The swirl effect of the liquid also assists the liquid breakup process after liquid emerges from the orifice.

The formation of a central air core is the most important phenomenon in a simplex nozzle. The size of the air core determines the effective flow area at the discharge orifice and thus controls the coefficient of discharge, which is one of the important performance parameter of the nozzle. Another important performance parameter is the spray cone angle that determines the coverage and dispersion of spray in the surrounding environment. As the mass flow rate through the atomizer is increased from zero, the performance parameters change sharply at first, but eventually at high mass flow rate, the discharge coefficient, the film thickness, and the spray cone angle become insensitive to the variations of flow Reynolds number. This indicates that discharge coefficient, cone angle and air core radius do not depend on injection pressure and keep constant values [2]. As mentioned above two important performance parameters in pressure-swirl atomizers are the discharge coefficient and spray cone angle. The discharge coefficient is the ratio of the actual to the maximum theoretical flow rate that is determined from the measured pressure drop across the atomizer.

$$
C_{d}=\frac{\dot{M}_{L}}{\rho_{L} A_{o} \sqrt{2 \Delta P / \rho_{L}}}
$$

where $\dot{M}_{L}, \rho_{L}, A_{o}$ and $\Delta P$ are the mass flow rate of injection liquid, the density of injection liquid, orifice area and the pressure drop along the injector respectively. The spray cone angle was calculated by:

$\theta=2 \tan ^{-1}\left(\frac{\bar{W}_{e}}{\bar{U}_{e}}\right)$

where $\bar{W}$ and $\bar{U}_{e}$ are the average swirl and axial velocities at the orifice exit.

Due to the difficulties outlined earlier, the initial investigations of simplex atomizer modeled the liquid as inviscid and the flow irrotational [3, 4]. Taylor [5] gave the most valid and pioneering theoretical treatment for potential flow in a swirl nozzle and predicted that the air core diameter and spray cone angle were inverse functions. Doumas and Laster [6] have reported an experimental study of such nozzles, measuring the discharge coefficient and the spray cone angle for more than 60 swirl atomizers covering a range of internal dimensions. They have developed zero dimensional models where fluids were assumed inviscid. In 1986 Rizk and lefebver [7] have investigated the internal flow characteristics of simplex swirl atomizers. The effects of the individual swirl atomizer geometrical dimensions on the thickness of annular liquid film at the nozzle exit and the effects of the fluid properties on the values of the discharge coefficient, the spray angle and the liquid film thickness, were studied. Horvay et al $[8,9]$ studied the velocity profiles within a pressure swirl atomizer. Their experiments were conducted using three different convergence configurations. Dumouchel et al [10] studied the two-dimensional viscous flow inside a pressureswirl atomizer by numerically solving the streamfunction and vorticity equations. They have used the Bloor and Ingham [11] analysis for the boundary-layer flow above a flat disk and they estimated the proportion of the fluid that enters the orifice through the boundary layer. Benjamin et al [12] have investigated the effects of various geometric and flow parameters on the performance of large-scale pressure swirl atomizers using optical methods. In 2001 Hansen et al [13] simulated the Newtonian fluid flow in a scaled model of a pressure-swirl atomizer via commercially available CFX-4.3 code. The Volume of Fluid (VOF) method was used to track the liquid-gas interface. Ma [14] has studied the internal flow characteristics in the swirl chamber for both large-scale and medium-scale pressure swirl atomizers. The internal flow field was measured using a two-color PIV system and refractive index matching fluids method. The spray cone angle and liquid film thickness of the nozzle were also obtained from the PIV image. Many numerical simulations have been conducted such as Yule and Chinn [15] and Datta et al [16]. Yule and Chinn [15] conducted a numerical study by treating the entire computational domain as single phase, and then guessing the interface by joining grid points where pressure is found to be atmospheric. In spite of large number of studies on the subject of Newtonian fluid inside pressure swirl injector only a few studies has been conducted with non-Newtonian fluid flow. Som et al $[17,18]$ determined both theoretically and experimentally the discharge coefficient and spray cone angle of swirl nozzle using a time-independent purely viscous powerlaw non-Newtonian fluid. In their papers, theoretical predictions were made through an approximate analytical solution of the hydrodynamics of flow inside the nozzle and experiments were carried out with aqueous solutions of CMC powder of various concentrations as the working fluid. Xue [18] developed two-dimensional axisymmetric computational fluid dynamics (CFD) model based on the ArbitraryLagrangian-Eulerian (ALE) method to predict the nonNewtonian flow in pressure-swirl atomizers. In 2007 Mandal [19] used FLUENT commercial code to predict the nonNewtonian fluid flow inside pressure-swirl atomizer. In Xue[19] and Mandal[20] simulation the flow inside pressureswirl atomizers assumed to be laminar in spite of this fact that the nature of the flow within pressure-swirl atomizer is turbulent. 
In this study by using power-law equation to calculate shear stress terms in the Navier-Stokes equations, the numerical model is used to compute Newtonian and non-Newtonian fluid flow inside the atomizers. A two-dimensional axisymmetric swirl model based on the VOF method was developed to predict the non-Newtonian flow within pressure-swirl atomizers. The code is also extended with the k-epsilon model for including turbulent effect. The time-independent purely viscous power-law fluids flow in pressure-swirl atomizers was simulated. The effects of shear-thinning fluids $(0.5<\mathrm{n}<1)$, viscous Newtonian $(\mathrm{n}=1)$ fluids and shear thickening fluids $(1<\mathrm{n}<1.5)$ have been considered on atomizer performance. The numerical results show that in constant pressure drop across the atomizer with increase the power-law index the tangential velocity decreases dramatically at the orifice exit but both the radial and the axial velocites decreases slightly. But the magnitude of swirl velocity decreases more than the magnitude of axial velocity and it leads to decrease in the spray cone angle.

\section{NOMENCLATURE}

$A_{o} \quad$ Orifice area

$A_{p} \quad$ Total inlet slot area

$D_{p} \quad$ Inlet port diameter

$C_{d} \quad$ Discharge coefficient

$\theta \quad$ Spray cone angle

$K \quad$ Atomizer constant

$D_{s} \quad$ Swirl chamber diameter

$d_{0} \quad$ Orifice hole diameter

$L_{s} \quad$ Swirl chamber length

$l_{o} \quad$ Orifice length

$P \quad$ Static pressure

$Q \quad$ Volume flow rate

$r \quad$ Radial coordinate

$x \quad$ Axial coordinate

$u \quad$ Axial velocity component

$v \quad$ Radial velocity component

$w \quad$ Swirl velocity component

$\rho \quad$ Density

$\alpha_{1} \quad$ Volume fraction of phase 1

$\vec{u} \quad$ Velocity vector

$G_{k} \quad$ Turbulence kinetic energy due to velocity gradients

$n \quad$ Power-law index

$m \quad$ Fluid consistency

$\tau \quad$ Viscous stress

$\mu \quad$ Dynamic viscosity

$\eta_{o} \quad$ Absolute coefficient of liquid viscosity

$\sigma_{i j} \quad$ Surface tension coefficient

\section{GOVERNING EQUATIONS}

Numerical Simulations of the unsteady two-phase flow field in high pressure swirl injector are governed by the continuity equation (Eq. 3) and Navier-Stokes equations (Eq. 4).

$$
\begin{aligned}
& \frac{\partial \rho}{\partial t}+\nabla(\rho \vec{u})=0 \\
& \frac{\partial(\rho \vec{u})}{\partial t}+\nabla(\rho \vec{u} \vec{u})=-\nabla p+\nabla\left[\mu\left(\nabla \vec{u}+\nabla \vec{u}^{T}\right)\right]+\rho \vec{g}+F_{v o l}
\end{aligned}
$$

The generalized Newtonian fluid base on Bird's model [21] is:

$$
\tau=-\eta\left(\nabla \vec{u}+(\nabla \vec{u})^{t}\right)=-\eta \dot{\gamma}
$$

According to the two-parameter power-law model:

$$
\eta=m \dot{\gamma}^{n-1}
$$

Where $\mathrm{n}$ is the power index (fluid behavior index) and $\mathrm{m}$ is the fluid consistency index. So the shear stress tensor can be expressed as,

$$
\tau=-m \dot{\gamma}^{n}
$$

The fluid is shear-thinning non-Newtonian for $\mathrm{n}<1$; Newtonian fluid for $\mathrm{n}=1$, is; and shear-thickening fluid for $\mathrm{n}>1$. In spite of the presence of the swirl chamber that creates a threedimensional flow configuration, in this paper due to long time that required for three-dimensional simulation, the twodimensional axisymmetric swirl model was used to compute flow through the injector. Due to the important role of the swirl velocity in flow inside the injector, the tangential momentum equation for two-dimensional swirling flows added to the momentum equations and it can be written as following:

$$
\begin{aligned}
\frac{\partial}{\partial t}(\rho w)+\frac{1}{r} & \frac{\partial}{\partial x}(r \rho u w)+\frac{1}{r} \frac{\partial}{\partial r}(r \rho v w)= \\
& \frac{1}{r} \frac{\partial}{\partial x}\left[r \mu \frac{\partial w}{\partial x}\right]+\frac{1}{r^{2}} \frac{\partial}{\partial r}\left[r^{3} \mu \frac{\partial}{\partial r}\left(\frac{w}{r}\right)\right]-\rho \frac{v w}{r}
\end{aligned}
$$

where $\mathrm{x}$ is the axial coordinate, $r$ is the radial coordinate, $u$ is the axial velocity, $v$ is the radial velocity, and $w$ is the swirl velocity. The VOF method was used to compute the volume fraction of each of the fluids in each computational cell throughout the domain. In the VOF method, the volume fraction of the first fluid in the cell is denoted as, $\alpha=0$ for an empty cell; $\alpha=1$ for a full cell and for partially filled with liquid, $\alpha$ has a value between zero and one. In order to track the position of a free surface between two different phases additional advection equation (Eq. 3) for the additional phase was solved. 
$\frac{\partial \alpha_{i}}{\partial t}+\vec{u} . \nabla \alpha_{i}=S$

where " $S$ " is the appropriate cavitation mass transfer sink or source term. Due to neglecting the cavitation phenomenon in this study the" $S$ "is considered to be equal to zero. Equation (4) is dependent on the volume fractions of phases through the properties $\rho$ and $\mu$. The properties of the first and the second fluids are calculated using:

$\rho=\sum_{i=1}^{n} \alpha_{i} \rho_{i}$

$\mu=\sum_{i=1}^{n} \alpha_{i} \mu_{i}$

where $\sum_{i=1}^{n} \alpha_{i}=1$

The velocity differences between the two phases in this model are not pronounced so the shared-field approximation can be safely used without adversely affecting velocity computations near the interface. The continuum surface force (CSF) proposed by Brackbill et al [22] is used for the surface tension model. The additional surface tension model for the VOF calculation results in a source term in equation (4) and is expressed as a volume force as follows:

$$
F_{v o l}=\sigma_{i j} \frac{\rho k_{i} \alpha_{i}}{(1 / 2)\left(\rho_{i}+\rho_{j}\right)}
$$

where $k_{i}$ is the curvature of the interface and $\sigma_{i j}$ is the surface tension coefficient.

The $k-\varepsilon$ renormalization group ( $\mathrm{RNG}$ ) model was used in order to calculate turbulence effect. The RNG-based $k-\varepsilon$ turbulence model is derived from the instantaneous Navier-Stokes equations, using a mathematical technique. Transport Equations for the RNG $k-\varepsilon$ Model are as follows:

$$
\begin{aligned}
& \frac{\partial}{\partial t}(\rho k)+\frac{\partial}{\partial x_{i}}\left(\rho k u_{i}\right)= \\
& \frac{\partial}{\partial x_{j}}\left(\alpha_{k} \mu_{e f f} \frac{\partial k}{\partial x_{j}}\right)+G_{k}+G_{b}-\rho \varepsilon-Y_{M}+S_{k} \iota \\
& \frac{\partial}{\partial t}(\rho \varepsilon)+\frac{\partial}{\partial x_{i}}\left(\rho \varepsilon u_{i}\right)=\frac{\partial}{\partial x_{j}}\left(\alpha_{\varepsilon} \mu_{e f f} \frac{\partial \varepsilon}{\partial x_{j}}\right)+ \\
& C_{1 \varepsilon} \frac{\varepsilon}{k}\left(G_{k}+C_{3 \varepsilon} G_{b}\right)-C_{2 \varepsilon} \rho \frac{\varepsilon^{2}}{k}-R_{\varepsilon}+S_{\varepsilon}
\end{aligned}
$$

where $G_{k}$ represents the generation of turbulence kinetic energy due to the mean velocity gradients, $G_{b}$ is the generation of turbulence kinetic energy due to buoyancy. The quantities $\alpha_{k}$ and $\alpha_{\varepsilon}$ are the inverse effective Prandtl numbers for $k$ and $\varepsilon$, respectively. $S_{k}$ and $S_{\varepsilon}$ are source terms. The model constants are $C_{1 \varepsilon}$ and $C_{2 \varepsilon}$ and they were assumed 1.42 and 1.68 respectively [23]. Using the RNG model causes to better handle low-Reynolds-number and near-wall flows .Turbulence, in general, is affected by swirl in the mean flow. The RNG model provides an option to account for the effects of swirl or rotation by modifying the turbulent viscosity appropriately.

\section{NUMERICAL METHOD}

In the numerical simulation the second order upwind scheme was employed to discrete momentum equations and the momentum equations solved implicitly. Also the SIMPLE algorithm substitutes the flux correction equations into the discrete continuity equation to obtain a discrete equation for the pressure correction in the cell. The implicit scheme was employed to discrete VOF equation. Since this equation requires the volume fraction values at the current time step a standard scalar transport equation is solved iteratively for each of the secondary-phase volume fractions at each time step. In this numerical simulation the volume fraction equation was solved once for each time step. This means that the convective flux coefficients appearing in the other transport equations will not be completely updated in each iterate, since the volume fraction fields will not change from iteration to iteration. In order to track better interface of fuel-gas inside the injector the modified HRIC was used to discrete VOF equation. The modified HRIC scheme is a composite Normalized Variable Diagram (NVD) scheme that consists of a non-linear blend of upwind and downwind differencing. To compare the computational results with experimental measurements, the assumption of axisymmetry requires determination of an equivalent "annular" inlet slot instead of the finite number of slots present in the real atomizer. The width of the "annular" slot as well as the radial and tangential velocities at the inlet are calculated by equating the angular momentum, total mass flow rate, and the kinetic energy of the liquid at the inlet ports with those in the experiments. The inlet boundary condition was applied to the top of the swirl chamber of injector. In order to specify the boundary conditions on the inlet the radial and swirl components of the velocity must be calculated from (Eq. 15).

$W_{\text {inlet }}=\frac{Q}{A_{p}} \cdot \frac{D_{s}-D_{p}}{D_{s}}$ and $V_{\text {inlet }}=\sqrt{\left(\frac{Q}{A_{p}}\right)^{2}-W_{\text {inlet }}^{2}}$

where $W_{\text {inlet }}, Q, D_{s}, D_{p}, V_{\text {inlet }}$ are mean tangential velocity at inlet, mass flow rate, diameter of swirl chamber, inlet port diameter (inlet port assume to be annular), mean radial velocity at inlet respectively.

\section{CODE VERIFICATION AND MESH STUDY}

In order to obtain reliable and accurate results, it is important to choose appropriate grid nodes. In this paper to validate the code, numerical results were compared with Ma's experimental data [13] (see table 2 and 3). In the numerical simulations the injection liquid assumed to be water at constant temperature of $300 \mathrm{~K}$. It is essential to validate the code with 
grid independence computational domain. For this reason the case 3 (see table 1)was selected and several number of grid nodes $(4500,9300$ and 18000) were considered for mesh study. The values of discharge coefficient and spray cone angle were compared for different grid numbers. For 9300 and higher values of this grid nodes the discharge coefficient and spray half of angle did not change significantly and kept constant values. The differences in the results for using two different grid nodes were very small and this indicates that 9300 grid nodes is sufficient to get grid-independent results.

Table 1: VARIATION OF CHARACTRISTIC GEOMETRY PARAMETERS

\begin{tabular}{|l|c|c|c|c|}
\hline & Case-1 & Case-2 & Case-3 & Case-4 \\
\hline Inlet area $\left(\mathrm{mm}^{2}\right)$ & 203 & 406 & 203 & 203 \\
\hline Exit orifice diameter(mm) & 21.6 & 28.8 & 28.8 & 21.6 \\
\hline Exit orifice length(mm) & 36.7 & 40.5 & 40.5 & 36.7 \\
\hline Swirl Cham. diameter(mm) & 76 & 76 & 76 & 76 \\
\hline Swirl chamber length(mm) & 38 & 38 & 38 & 89 \\
\hline Mass flow rate(Kg/s) & 0.5 & 0.95 & 0.95 & 0.63 \\
\hline
\end{tabular}

Table 2: COMPARISION BETWEEN MA'S EXPERIMENTAL DATA AND NUMERICAL RESULTS

\begin{tabular}{|l|l|l|l|l|}
\hline & Case-1 & Case-2 & Case-3 & Case-4 \\
\hline & \multicolumn{5}{|c|}{ Spray cone half angle (deg), $\theta$} \\
\hline CFD-1st order upwind & 38.33 & 43.32 & 47.96 & 39.25 \\
\hline CFD-2nd order upwind & 40.20 & 39.47 & 45.94 & 39.38 \\
\hline Experiment & 42.4 & 38.5 & 45.0 & 38.5 \\
\hline & \multicolumn{5}{|c|}{ Discharge coefficient, $C_{d}$} \\
\hline CFD-1st order upwind & 0.1750 & 0.214 & 0.1311 & 0.1481 \\
\hline CFD-2nd order upwind & 0.1701 & 0.205 & 0.1293 & 0.1464 \\
\hline Experiment & 0.17 & 0.20 & 0.12 & 0.16 \\
\hline
\end{tabular}

Figure 2 shows an axisymmetric contour of volume fraction for case 3. This figure also shows the boundary condition used for the simulation.

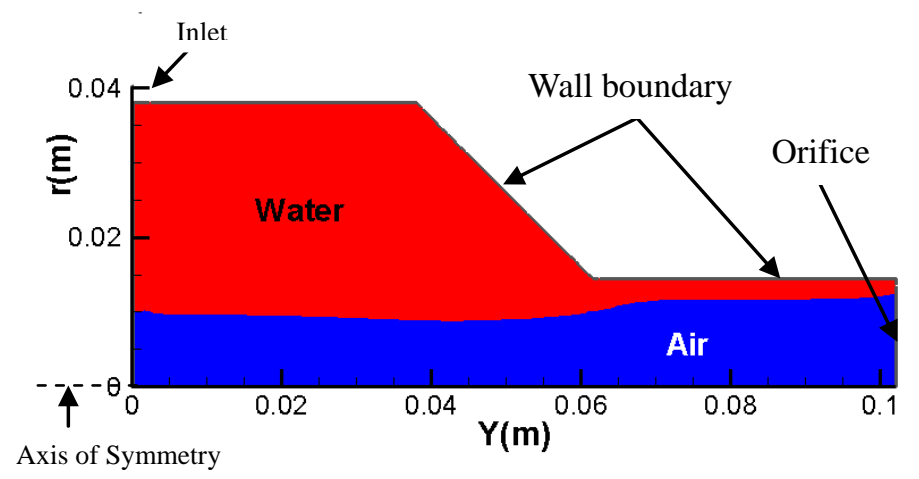

Figure 2: AXISYMITRIC CONTOUR OF VOLUME FRACTION FOR CASE 3

\section{RESULTS AND DISCUSION}

Case 3 was selected and the effect of variation in power-law index on the performance of pressure-swirl atomizers has been considered. The effects of shear-thinning fluids $(0.5<\mathrm{n}<1)$, viscous Newtonian $(\mathrm{n}=1)$ fluids and shear thickening fluids $(1<\mathrm{n}<1.5)$ on atomizer performance (discharge coefficient and spray cone angle) are numerically investigated. Figure 3 shows axisymmetric contours of velocity magnitude (right) and velocity stream lines (left) for fluid with 0.4 as power-law index and the fluid with 1.4 power-law index. The streamlines are based on the axial and the radial component of velocity and do not include the swirl component of velocity which is perpendicular to the plane of the figure. Due to the viscosity it is clear that for shear-thinning fluid with $n=0.4$ the magnitude of velocity higher than shear thickening fluid. Large recirculating vortex structures are present in the swirl chamber in these two cases, however, their numbers, sizes, and locations are significantly different. The bulk of vorticity for shear thinning fluid is bigger than the bulk of vorticity for shear thickening fluid due to viscosity effect.

Larger value of $\mathrm{n}$ corresponds to increase the viscosity and results in an increasing decay of swirl energy. Figures 4, 5 and 6 demonstrate the variation of axial velocity, swirl velocity and radial velocity at exit orifice radius for different power-law indices $\mathrm{n}=0.4,0.6,1.0,1.2$ and $\mathrm{n}=1.4$. It is clear that by increasing the power-law index the axial swirl and radial velocities decrease. The amplitude of swirl velocity varies between $7 \mathrm{~m} / \mathrm{s}$ for $\mathrm{n}=0.4$ to $3 \mathrm{~m} / \mathrm{s}$ for 1.4 power-law index. But the variations of the axial and radial velocities are small for different power-law indices. As the flow enters the exit orifice section and is pushed along the orifice wall forming a thin liquid film, the radial component of velocity becomes negligible. At the exit orifice, the locations of the maximum axial and swirl velocities are more close to the wall than the interface. But for radial velocity the maximum occurs near the surface of liquid and air. Comparing to the axial velocities at the orifice, the average values become larger due to the decrease of the film thickness. The shear-thickening fluid has smaller velocity magnitude. Figures 7 and 8 show that trend of discharge coefficient and spray cone angle are approximately the same but the behavior of cone spray angle is in the opposite of discharge coefficient while the power- law index changes. Figure 7 shows that as power-law index increases from 0.4 to 1 , spray cone angle decreases slightly and as $\mathrm{n}$ changes from 1 to 1.4 , spray cone angle decreases dramatically. Theoretically, this is resulted also results from increasingly decaying swirl energy at the exit for a longer orifice length. As it can be seen the amplitude of half of spray cone angle is between $41^{\circ}$ to $51^{\circ}$. The trend of $n$ variation with spray cone angle is linear as well as the discharge coefficient. 

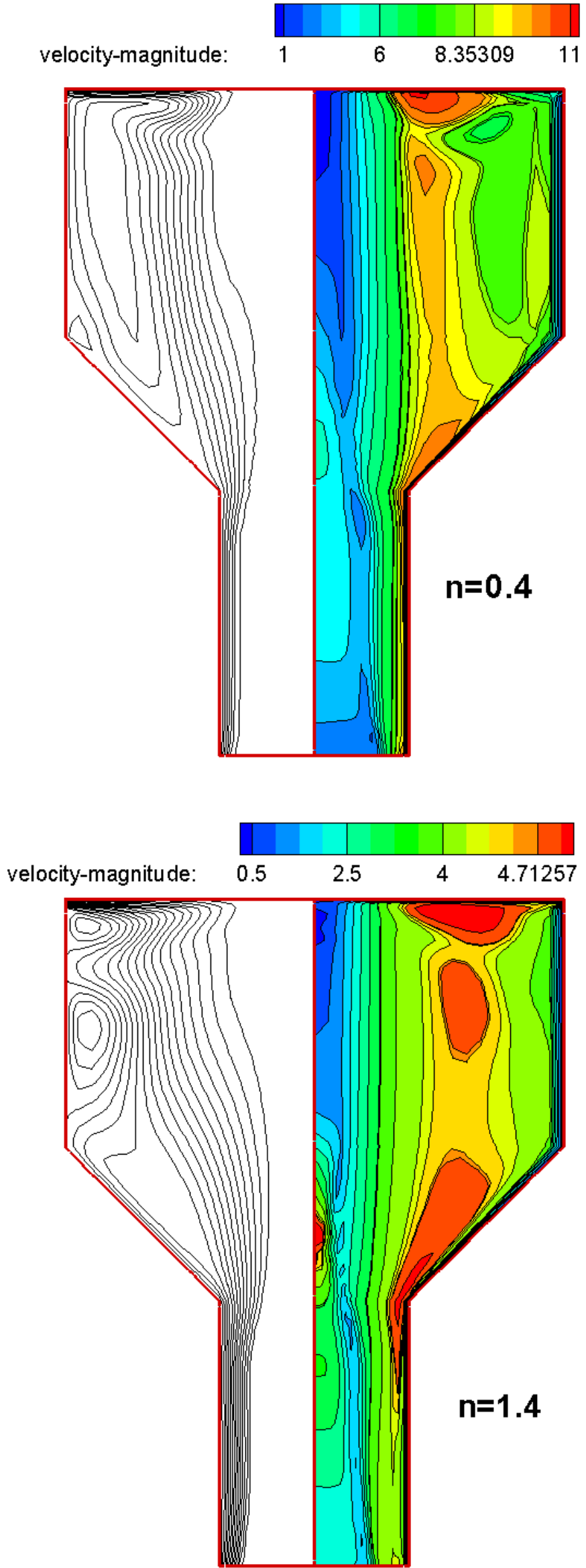

Figure3. AXSYMITRIC COUNTORS OF VELOCITY MAGNITUDE (RIGHT) AND VELOCITY STREAM FUNCTION FOR TWO DIFFERENT $n$ INDICES.

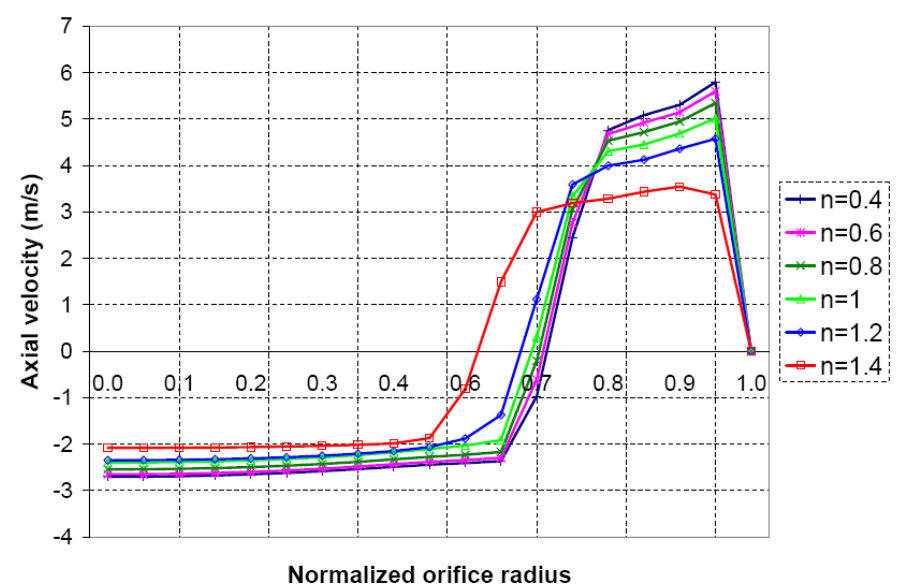

Figure 4: CALCULATED AXIAL VELOCITY FOR VARIOUS POWER-LAW INDICES (n) AT EXIT ORIFICE HOLE

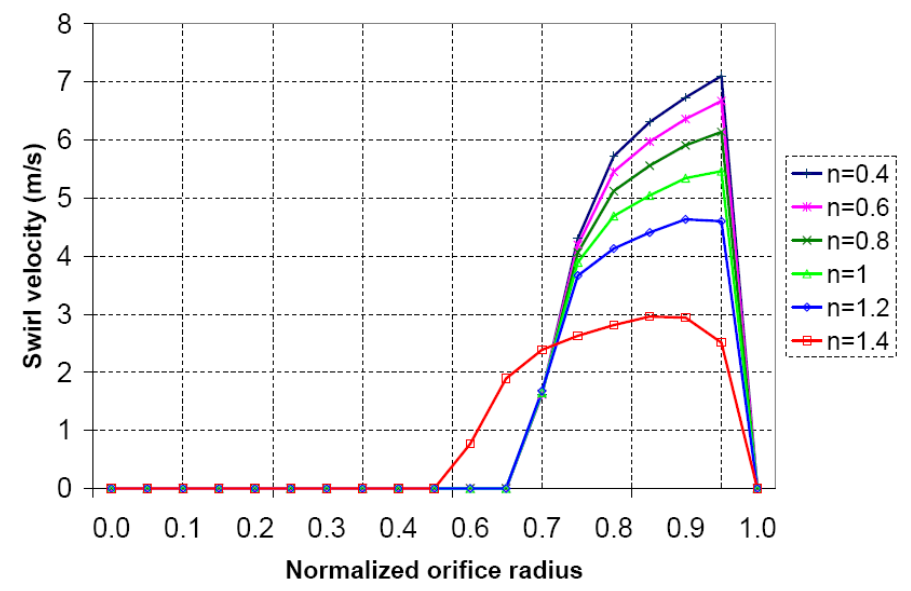

Figure 5: CALCULATED SWIRL VELOCITY FOR VARIOUS POWER-LAW IINDICES (n) AT EXIT ORIFICE HOLE

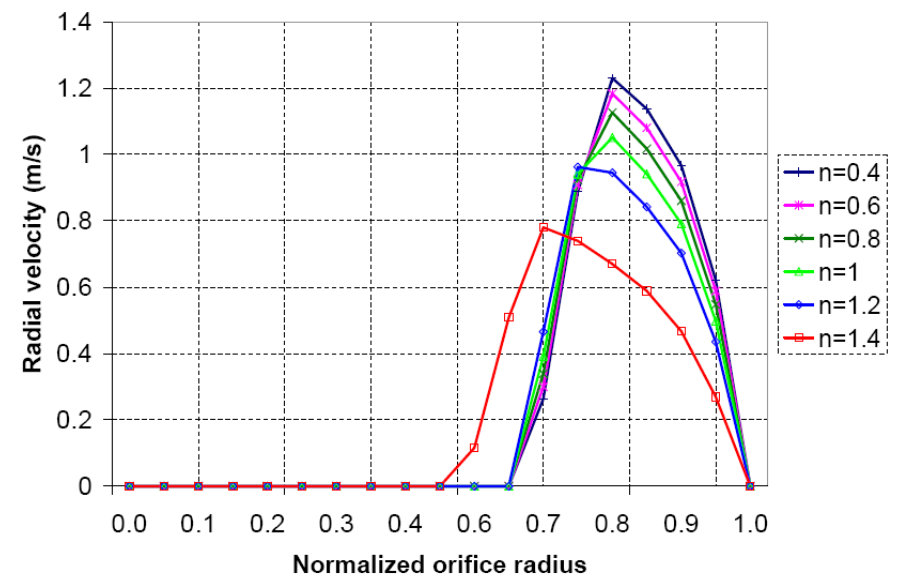

Figure 6: CALCULATED RADIAL VELOCITY FOR VARIOUS POWER-LAW INDEX (n) AT EXIT ORIFICE HOLE E OR HOLE 


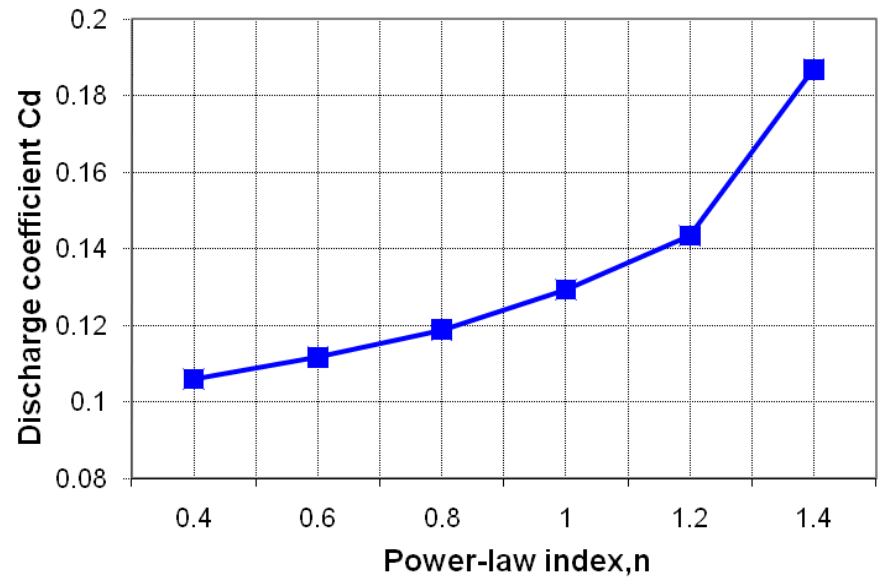

Figure 7: DISCHARGE COEFFICIENT VS POWER-LAW $\operatorname{INDEX}(\mathrm{n})$

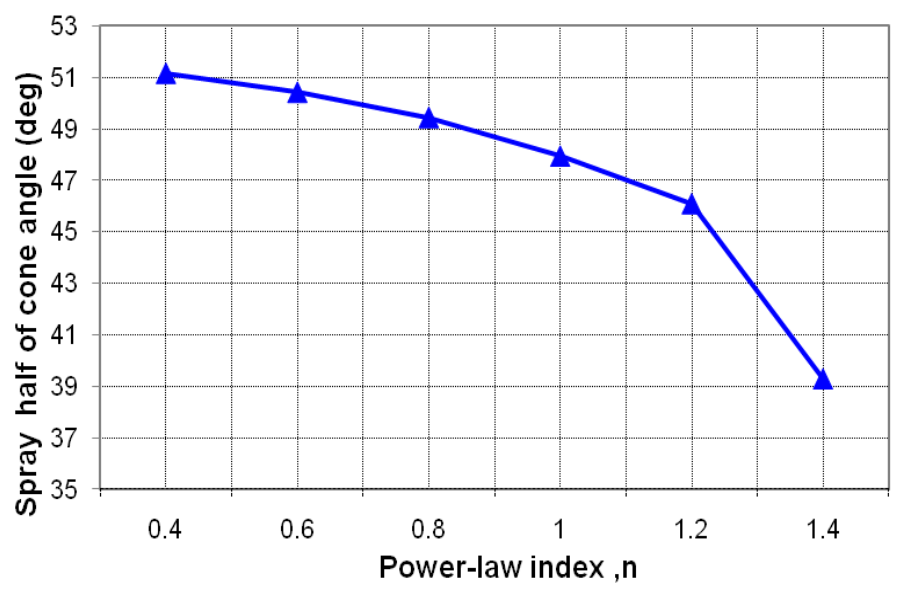

Figure 8: SPRAY CONE HALF ANGLE VS POWER-LAW $\operatorname{INDEX}(n)$

\section{CONCLUSIONS}

In this study by using power-law equation to calculate shear stress terms in the Navier-Stokes equations, the numerical model is used to compute Newtonian and non-Newtonian fluid flow inside the atomizer. A two-dimensional axisymmetric model based on the VOF method was developed to predict the non-Newtonian flow within pressure-swirl atomizers. The timeindependent purely viscous power-law fluids flow in pressureswirl atomizers was simulated. The effects of shear-thinning fluids $(0.5<\mathrm{n}<1)$, viscous Newtonian $(\mathrm{n}=1)$ fluids and shear thickening fluids $(1<\mathrm{n}<1.5)$ have been considered on atomizer performance. The numerical results show that in constant pressure drop across the atomizer by increasing the power-law index the tangential velocity decreases dramatically at the orifice exit and both the radial and the axial velocities decreases slightly. But the magnitude of swirl velocity decreases more than decreasing in magnitude of the axial velocity and it leads to decrease in the spray cone angle. On the other hand due to increase in shear stress with increasing the power index the film thickness increases at the orifice exit and the discharge coefficient increases. Increasing in film thickness leads to decrease the spray cone angle.

\section{REFERENCES}

[1] Ibrahim, A., 2006, "Comprehensive Study of Internal Flow Field and Linear and Nonlinear Instability of an Annular Liquid Sheet Emanating from an Atomizer" PhD Thesis, Department of Aerospace Engineering and Engineering Mechanics, University of Cincinnati.

[2] Lefebvre, A. H., 1989, Atomization and Spray, Hemisphere Publishing Co.

[3] Giffen, E., and Muraszew, A., "The Atomization of Liquid Fuels, John Wiley, 1953.

[4] Novikof, I. I.,1948 “ Atomization of Liquids by Centrifugal Nozzles, J. Tech. Phys., Vol. 18, no. 3, p. 345.

[5] Taylor, G.I., 1948, “The Mechanics of a Swirl Atomizer, in: Proceedings of the Seventh International Congress Applied Mechanics, vol. 2, no. 1, pp. 280.

[6] Doumas, M., Laster, R. 1953,'Liquid-Film Properties for Centrifugal Spray Nozzles" Chemical Engineering Progress, pp.518-526.

[7] Rizk, N. K. And Lefebvre, A. H., 1986, "Influence of Liquid Properties on the Internal Flow Characteristics of Simplex Swirl Atomizers, Atomization and Spray Technology, Vol. 2, No. 3, May-June, pp 219-233.

[8] Horvay, M. And Leuckel, W., 1985, "LDA-Measurements of Liquid Swirl Flow in Converging Swirl Chambers with Tangential Inlets", Proc. 2nd Int. Symp. LDA Lisbon. Paper 14.

[9] Horvay, M. And Leuckel, W., 1986, "Experimental and Theoretical Investigation of Swirl Nozzles for Pressure-Jet Atomization", German Chem. Eng., Vol. 9, pp 276-283.

[10] Dumouchel, C., Bloor, M., Dombrowski, N., Ingham, D. 1993,"Viscous Flow in a Swirl Atomizer" Chemical Engineering Science, vol. 48, no. 1, pp. 81- 87.

[11] Bloor, M. I. G. and Ingham, D. B., , 1977 "Axially Symmetric Boundary Layer on a Finite Disk", Phys. Fluids, vol. 20, no. 8, p. 1228.

[12] Benjamin, M. , Mansour, A., Samant, U. G., Jha, S., Liao, Y., Harris, T. and Jeng, S. M., 1998 "Film Thickness, Droplet Size Measurements and Correlations for Large Pressure- Swirl Atomizers", American Society of Mechanical Engineers, Paper 98-Gt-537,

[13] Hansen, K. G., Madsen, J., Trinh, C. M., Ibsen, C. H., Solberg, T. and Hjertager, B. H., 2002 "A computational and Experimental Study of the internal Flow In a scaled Pressure-swirl Atomizer" ILASS-Europe, Zaragoza,

[14] Ma, Z., 2001 "Investigation on the Internal Flow Characteristics of Pressure-swirl Atomizer" Ph.D. Thesis, University Of Cincinnati, Department of Aerospace Engineering and Engineering Mechanics of the College of Engineering,

[15] Yule, A. J., and Chinn, J. J. 1997, "Pressure Swirl Atomizer Internal flow and Performance", Proceedings of the 10th Annual conference on Liquid Atomization and Spray Systems, Inst. For Liquid Atomization and Spray Systems-Americas, Irvine, CA, pp.205-209.

[16] Datta, A. and Som S. K., 2000, "Numerical Prediction of Air Core Diameter Coefficient of Discharge and Spray Cone Angle of a Swirl Spray Pressure Nozzle", Int. J. Heat Fluid Flow, vol. 21, pp. 412.

[17] Som, S. K., 1983, "Theoretical and Experimental Studies on the Coefficient of Discharge and Spray Cone Angle of a Swirl Spray Pressure Nozzle Using a Power-Law Non- 
Newtonian Fluid", Journal of Non-Newtonian Fluid Mechanics, 12, pp 39-68.

[18] Som, S. K. and Biswas, G., 1986 ,Coefficient of Discharge and Spray Cone Angle of a Pressure Nozzle with Combined Axial and Tangential Entry of Power-Law Fluids", Applied Scientific Research, 43, pp 3-22.

[19] Xue, J., 2001, "Computational Simulation of Flow Inside Pressure-Swirl Atomizers", Ph.D. Thesis, Department of Aerospace Engineering and Engineering Mechanics, University of Cincinnati,

[20] Mandal, A., 2007, "Computational Modeling of Non Newtonian Fluid Flow in Simplex Atomizers" M.Sc.
Thesis, Department of Aerospace Engineering and Engineering Mechanics, University of Cincinnati.

[21]Bird, R. B., Stewart, W. E., and Lightfoot, E. N., 2002, Transport Phenomena, Second Edition, John Wiley Publishing.

[22] Brackbill, J. U., Kothe, D. B., and Zemach, C., 1992 “A Continuum Method for Modeling Surface Tension”, $J$. Comput. Phys., vol. 100, pp. 335-354.

[23] Lam, S., 1992,"On The RNG Theory of Turbulence" Journal of Physics of Fluid, A, 4, pp.1007-1017. 